\title{
XXVIII. The electrical effect of the ultra-violet spectrum
}

\author{
E.W.B. Gill B.A. B.Sc.
}

To cite this article: E.W.B. Gill B.A. B.Sc. (1910) XXVIII. The electrical effect of the ultra-violet spectrum , Philosophical Magazine Series 6, 19:110, 290-300, DOI: 10.1080/14786440208636801

To link to this article: http://dx.doi.org/10.1080/14786440208636801

曲 Published online: 21 Apr 2009.

Submit your article to this journal $\sqsubset x$

Џll Article views: 5

Q View related articles $₫$ 
first half-oscillation the metallic streamers are more intense at the electrode which is for the instant negative.

4. The velocity of the metallic vapour has been measured from the slope of the first edge of the initial streamer. The velocity falls off with increase of self-induction; but it was possible to show that this is due to a reduced temperature.

5. Two simultaneous velocities in one and the same spectral line are seen in the case of calcium vapour.

I wish to express my indebtedness to Prof. Schuster and to Prof. Rutherford for their interest in these experiments and for many suggestions.

Physical Laboratory,

Manchester University.

XXVIII. The Electrical Effect of the Ultra-violet Spectrum. By E. W. B. GinL, B.A., B.Sc.; Fellow of Merton College, Oxford* ${ }^{*}$.

WHEN ultra-violet light falls upon a metal plate it is well known that negative ions are set free at the surface of the plate, the effect being most marked in the case of zinc. The present investigation was undertaken to ascertain the relative powers of each part of the ultra-violet spectrum for setting free negative ions.

The existence of ultra-violet light was first discovered by its acting upon silver chloride, and most of our knowledge of the ultra-violet spectrum has been obtained by photographing it. The discovery was soon made that glass was opaque to these rays, and it was found necessary to use quartz, or better still fluorite, for the prisms and lenses used in producing the spectrum. The early photographic work consisted simply in detecting various lines in the ultra-violet spectrum, and it was not till gratings were employed that the wavelengths of these lines were determined. If the spectrum be produced by a quartz prism, the absorption of rays by the quartz and also by the air gets very considerable as the waves get shorter. Schumann $\dagger$ investigated the absorptive power of quartz and found that a plate $2 \mathrm{~cm}$. thick cut off all wave-lengths shorter than $185 \mu \mu$.

For the purposes of this investigation the most important measurements were those of Rubens $\ddagger$, who found for the

* Communicated by Prof. J. S. Townsend, F.R.S.

+ Wien. Ber. cii. II. A. p. 415 (1893).

\pm Wied. $A n n$. liv. p. 476. 
ordinary ray in quartz the refractive indices corresponding to different wave-lengths as far as $\lambda=200 \mu \mu$. His results are shown in fig. 1.

Fig. 1.

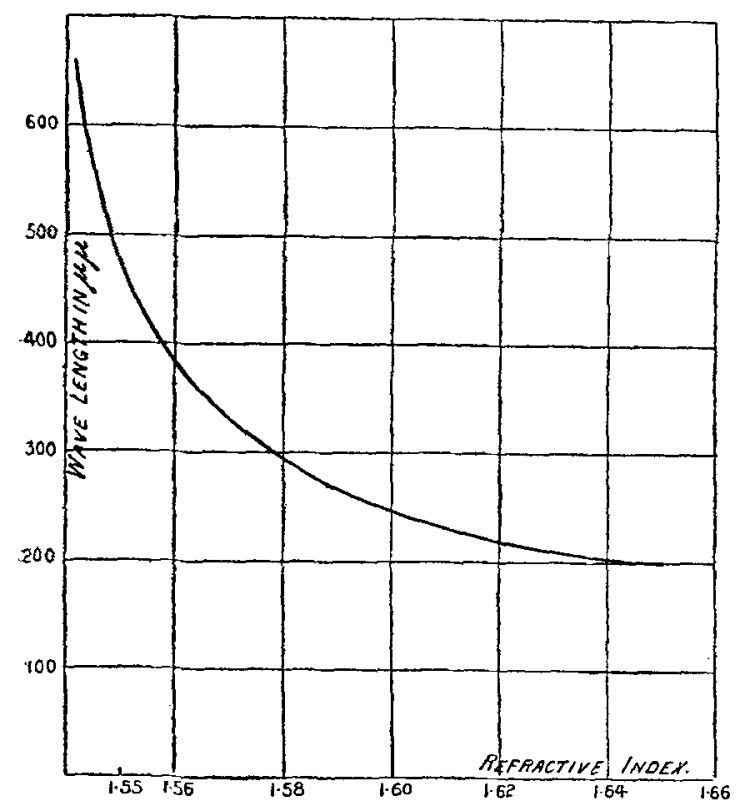

The ultra-violet spectrum has also been examined by the bolometer, and Pfliiger * has by this means measured approximately the energy of the different parts of the spectra of spark discharges between terminals of various metals. For aluminium terminals and a fluorspar prism he reached a limit of $\lambda=180 \mu \mu$. His results will be mentioned later.

A third and novel method of studying the ultra-violet spectrum is based on the well-known fact that ultra-violet light rays have a very destructive effect on various bacteria, and Professor Dreyer has very kindly given me the following account of experiments hased upon this. Dr. Sophus Bangt investigated the effect of various parts of the spectrum, and showed that the bactericidal power commenced just about the violet end of the spectrum, rose to a maximum at about $350 \mu \mu$, fell to a minimum at $310 \mu \mu$, and then rose very

* Annalen der Physik, xiii. p. 890 (1904).

† "Om Fordelingen af bakteriedrabende Stossler i Kulbuelysets Spektrum," Meddel. fra Finsens medic. Lysinstitut, ix. 1904, p. 123. 
quickly to a second maximum higher than the first at $300 \mu \mu$, and remained at that value without any decrease till $210 \mu \mu$, when a slight fall occurred to $200 \mu \mu$, where the observations terminated. Professor Dreyer *, however, made the interesting observation that by sensitizing the bacteria by erythrosin they could be made as sensitive to long as to short waves, in much the same way as a photographic plate can be made responsive to different rays.

A little consideration will show that there is not of necessity any intimate connexion between the photographic effect, the electrical effect, and the energy of any given portion of the spectrum. The photographic effect may be roughly estimated by the time of exposure required to photograph any part of the spectrum. The visible spectrum has a large effect upon a photographic plate, but as the wavelength gets shorter the effect gets less and less, and even if special plates (without gelatine, which Schumann found absorbed the shorter waves) be used very great difficulty is found in photographing as far as $\lambda=185 \mu \mu$, using a quartx prism and lenses. The electrical effect, on the other hand, is practically zero in the visible spectrum, the rays which set free negative ions being almost entirely cut off by glass, and, as will be shown later, the maximum electrical effect in the spectrum of a spark-discharge does not coincide with the rays which give the maximum photographic effect. In fact the electrical method for detecting the light is much more sensitive than the photographic for short waves.

The distribution of the energy also is independent of the intensities of either the photographic or the electricnl effects; thus the energy extends over the whole spectrum, and the electrical effect is confined to the ultra-violet; but it is a remarkable fact that Pflüger's energy curve reaches o maximum for the same rays as the curve giving the intensity of the electrical effect. The electrical curve, as will be seen later, has a second maximum, but unfortunately Pflüger's results do not reach as far as this.

In the electrical research to be described below the spectrum was formed by a quartz prism, and it may be useful to summarize the chief results obtained so far for the spectrum produced in this manner. Rubens' curve (fig. 1) shows that for a small range of spectrum $d n$ ( $n$ here and afterwards. denoting refractive index) the corresponding $d \lambda$ in the visible spectrum is large, but gets less as $n$ increases, and at

* "Sensibilisering af Mikroorganismer og dyriske Vœo," Meddel. frar Finsens medic. Lysinstitut, vii. $190 \mathrm{~s}$, p. 110. 
the furthest points $\frac{d n}{d \lambda}$ becomes very great for the large values of $n$, where the curve is nearly parallel to the axis of $\mu$. Beyond this point $(\lambda=200 \mu \mu)$ the connexion between $\lambda$ and $n$ is unknown, but quartz is said to cut off all waves shorter than $\lambda=185 \mu \mu$; this, however, is not in agreement with the results obtained by the electrical method. 'Rubens' curve is drawn for the ordinary ray, but after refraction through quartz the extraordinary ray is not very far separated from the ordinary, and is, moreover, very feeble in its effects. The absorption by quartz undoubtedly increases as the waves get shorter, as is also the case with the absorption by air.

The apparatus used to measure the electrical effects of the different parts of the spectrum was as follows.

The source of light was an oscillatory electrical discharge passed between two aluminium terminals; the length of the spark-gap was $4 \mathrm{~mm}$. and the terminals about $1 \mathrm{~mm}$. in diameter. The spark-gap was connected to the secondary of an 8-inch Ruhmkorff coil in parallel with a leyden-jar. The current through the primary was abont 3 amperes, and a hammer interrupter was used. This source of light, besides being very rich in ultra-violet rays, was of very steady intensity.

Fig. 2.

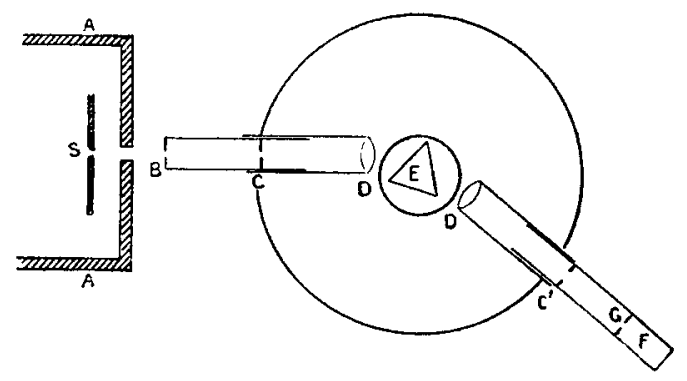

The spectrum was formed as in fig. 2. The spark-gap $\mathrm{S}$ was placed in a metal box $A$, this, as usual, being necessary to shield the rest of the apparatus from induction effects.

The light entering the collimator tube through a vertical slit at $B$ was rendered parallel by the first quartz lens $D$ and fell upon the prism $\mathrm{E}$; this prism was of quartz having angles of 60 degrees. With such a prism working at minimum deviation no trouble can be experienced from 
light internally reflected. The light, after passing through the prism, was brought to a focus by the second quartz lens. $D$, exactly similar to the first, at the vertical slit $G$, which was the entrance to the detector $F$ to be described later. It was found better in practice to have the spark-gap vertical and not horizontal as shown in the figure. The tubes carrying the lenses D, D were telescopic, allowing the lenses to be focussed for light of any refractive index within certain limits. The apparatus was mounted on a strong graduated spectroscope table which allowed the prism and the receiver to be rotated through any desired angle. The rays in travelling from the spark-gap $\mathrm{S}$ to the detector $\mathrm{F}$ traversed a distance of about $90 \mathrm{~cm}$. of air (at atmospheric pressure), and the central ray passed through about $3 \mathrm{~cm}$. of quartz, The tubes were blackened on the insides to prevent light being reflected from the sides, but as it seemed uncertain whether lampblack absorbed all the rays the sides of the tubes were cut away in places and two diaphragms $\mathrm{C}, \mathrm{C}^{\prime}$ inserted to stop oblique light.

\section{The Electrical Measurements.}

The light which entered the detector fell upon a zinc plate and caused a number of negative ions to be set free at its surface. The charge which thus escapes from the plate is very small since the slits at $G$ and $B$ (fig. 2) are narrow, so that in order to detect the effect of the light it is necessary to magnify the charge.

Professor Townsend has shown that if the zinc plate be arranged opposite and parallel to a second plate at distance $d$, and if the electric force between the plates is X, then if $n_{0}$ ions be set free at the zinc plate the total number of ions is reaching the positive plate is given by the equation

$$
n=n_{0} \frac{(\alpha-\beta) e^{(\alpha-\beta) d}}{\alpha-\beta e^{(\alpha-\beta) d}},
$$

where $\alpha$ and $\beta$ are functions of $\mathrm{X}$ and the pressure $p$ only. The multiplying factor $\frac{(\alpha-\beta) e^{(\alpha-\beta) d}}{\alpha-\beta e^{(\alpha-\beta) d}}$ can be made as large as we please by suitably adjusting $\mathrm{X}$ and $p$. During an experiment $p$ was kept at $4 \mathrm{~mm}$. of mercury, and $\mathrm{X}$ was about 700 volts per $\mathrm{cm}$. This gave a big factor and was not too near the sparking potential to produce irregularities. The charge transferred was measured by a quadrant electrometer, and the ratio of the readings of the electrometer gave in consequence the ratio of the numbers of ions set free for 
two different portions of the spectrum. The actual arrangement of the detector is shown in fig. 3. It was circular in

Fig. 3.

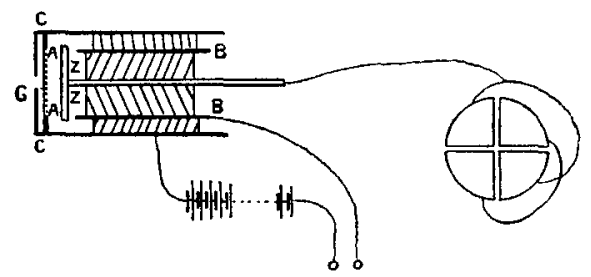

section except for the slit G, through which the light entered, which was vertical. The light having entered through a quartz plate CC passed through a silver wire gauze $\mathrm{AA}$ and fell upon the zinc plate $\mathrm{ZZ}$, distant $6 \mathrm{~mm}$. from the gauze. The plate ZZ was connected by a brass rod with the electrometer, and the silver gauze was kept at a potential of 400 volts by being connected through the outside brass tube to a battery of small storage-cells.

To prevent electrical leakage a tube BB kept constantly at zero potential was inserted. The shaded portions represent ebonite which was fastened by black wax to the brass tubes. All the joints were made air-tight and the apparatus was exhausted to a pressure of $4 \mathrm{~mm}$. through a tube (not shown in the figure).

The method of actually carrying out an experiment was as follows :- Suppose the effect of light of a given refractive index $n$ was to be measured. As the focal lengths of the lenses D, D (fig. 2) vary with the refractive index it was necessary to find the focal length corresponding to $n$ from the formula $f \propto \frac{1}{n-1}$ (the extreme values of $f$ were $36 \mathrm{~cm}$. for $n=1.54$ and $27.7 \mathrm{~cm}$. for $n=1 \cdot 71$, this being the range over which electrical effects were detected). The lenses were then focussed for parallel light for the particular value of $f$. All experiments were conducted at minimum deviation and the angles of incidence and deviation were calculated for this value of $n$ for minimum deviation; which gave the proper setting for the prism and receiver. With this arrangement the ray of refractive index $n$ is finally brought to a focus at the centre of the slit of the detector. In order to obtain a measurable effect it was found convenient to have this slit about 5 or $6 \mathrm{~mm}$. in width $(5.5 \mathrm{~mm}$. actually) and so other rays slightly out of focus were also gathered in on either side of the central ray. If the amount of spectrum 
entering the slit be the portion between $n+\frac{1}{2} d n$ to $n-\frac{1}{2} d n$ it is easy for the particular dimensions of the apparatus used to show that

$$
d n=\frac{n-1}{36} \sqrt{1-\frac{n^{2}}{4}} \text { approximately, }
$$

the lenses being properly focussed and the prism having an angle of $60^{\circ}$. The extreme observations were given by $n=1.56$ and $n=1 \cdot 71$, and the corresponding values of $d n$ were $\cdot 009$ and $\cdot 0105$; it is thus seen that $d n$ never differs much from 01 and as readings were taken for $n=1.56$, $1.57 \ldots 1.71$ the whole spectrum was embraced in these 16 readings. A few intermediate readings were also taken near points of interest on the resulting curve.

It must be borne in mind that although the range of spectrum $d n$ gathered in is approximately constant yet the number of different wave-lengths $d \lambda$ gathered in by the slit is by no means constant.

The apparatus being properly adjusted for minimum deviation for refractive index $n$ the light was turned on for 10 seconds and the corresponding deflexion of the electrometer noted. The prism was then tumed through some angle $\alpha$ to the position corresponding to some other index of refraction $n^{\prime}$ and the detector was turned through $2 \alpha$, thus being put in the minimum deviation position for $n^{\prime}$, the lenses were refocussed for refractive index $n^{\prime}$ and the deflexion again noted in 10 seconds. The ratio of the two readings gave the relative number of ions given off by the two ranges of spectra $n-\cdot 05$ to $n+\cdot 05$, and $n^{\prime}-\cdot 05$ to $n^{\prime}+\cdot 05$. Care must be taken lest in the second focussing we alter the cone of light received by the apparatus from the spark. To keep this constant $\mathrm{S}, \mathrm{B}$, and $\mathrm{C}$ (fig. 2) were kept fixed in space; and in order that the length of air traversed by the rays should be constant $G$ was kept at a fixed distance from the prism.

To prevent cumulative errors three standard positions were taken corresponding to values of $n$ of $1 \cdot 57,1 \cdot 61$, and $1 \cdot 65$ respectively, and the ratios of the numbers of ions set free at these very carefully determined. All other ratios were referred to one or other of these positions; for example, the effect at $n=1.59$ was compared with that at $n=1.61$.

\section{General Results.}

A large number of observations were made and the mean taken, the result being shown in the curve given in fig. 4. The method of construction of the curve will be understood 
from the following example; the ordinate at the point $n=1 \cdot 64$ represents in some arbitrary units the number of ions given off in 10 seconds by the zinc plate when light having

Fig. 4.

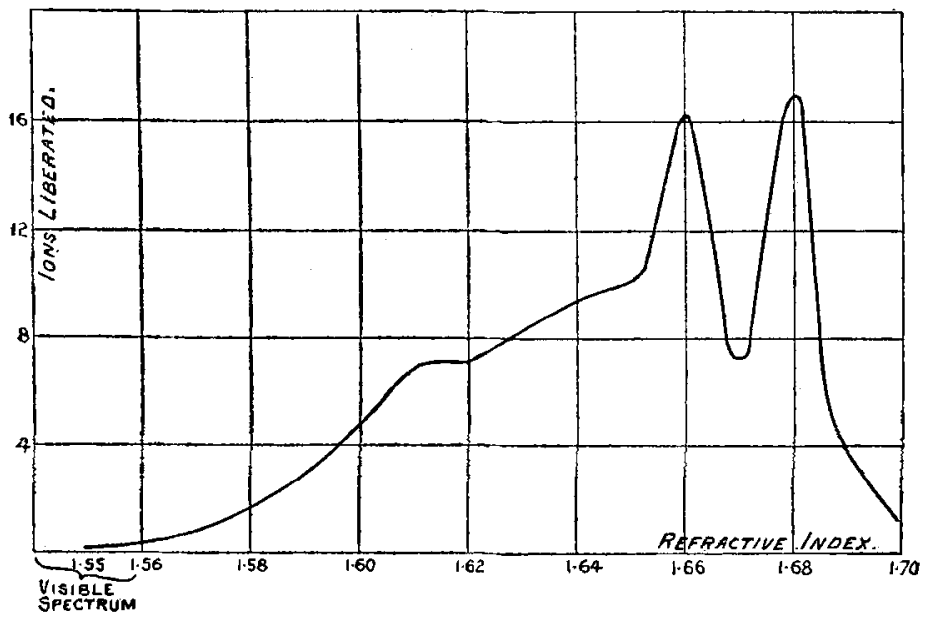

refractive indices between 1.635 and 1.645 fell upon it. The mnits are of necessity arbitrary because the actual number of ions given off is unknown. The limitations of the curve may be briefly recapitulated here. In the first place it simply refers to the spectrum produced by a quartz prism, no correction having been made either for the absorption of the quartz or of the air through which the rays passed, the absorption in both cases increasing with $n$. Secondly, in order to produce a measurable effect a comparatively large portion of the spectrum is included in each reading, and the curve gives no information about the effect of lines or narrow bands in the spectrum.

Thirdly it should be noticed that the curve gives the connexion between the number of ions set free and the refractive index. A curve giving the connexion between the number of ions liberated and the wave-length would be very different. Rubens' curve shows that $\frac{d \lambda}{d n}$ for the visible spectrum is very big, decreasing very rapidly as $n$ increases, and if therefore a curve connecting $n$ and $\lambda$ were drawn it would show that the effects of the small wave-lengths were very much more marked than even the effect of large values of $n$ in fig. 4. This curve has not, however, been drawn 
because it would have to be obtained by combining Rubens? curve (fig. 1) with fig. 4, and his measurements do not go as far as the electrical ones; it would be in addition slightly misleading because for any particular value of $n$ we have a mixture of two wave-lengths corresponding respectively to the ordinary and extraordinary rays, though for quartz these wavelengths are nearly equal and the extraordinary ray is of very feeble intensity.

The curve given represents the mean of a number of observations, but observations made at different times showed variations greater than experimental errors which have not yet been explained. There are three possible causes of variation, the state of the zine plate may vary, the light emitted by the spark may vary, or the absorption by the air may vary. Taking these in order it is true in the first place that a freshly polished zinc plate is very active in giving off ions, and as it gets dirty, i.e. oxidized, the number of ions given off (other things being equal) decreases, but a steady state is soon reached. The only thing which appears to disturb this steady state is the passace of a spark-discharge from the wire gauze to the zinc plate, but even then the original steady state is regained after 3 or 4 hours. In the second place the light from the spark-gap was kept as constant as possible by always having the same current through the primary, the same length of spark, and approximately the same adjustment of the hammer interrupter. On the whole it is improbable that either of the above canses is responsible for the variations, a supposition that is partly confirmed by the fact that on any one day the readings were consistent, the variation being from day to day; and it seems likely that the absorption by the air is the determining factor. The published results for air-absorption by different investigators. are very inconsistent, and it may be that the absorption is more due to the moisture in the air than to the air itself, which would explain the variation from day to day.

A study of the curve obtained (fig. 4) shows that the electrical effect is just beginning about the middle of the visible spectrum and increases up to a first maximum for refractive index $n=1 \cdot 66$, sinks to a minimam at $1 \cdot 67$, and reaches a second maximum for $n=1 \cdot 68$, rapidly decreasing after that. The position of the minimum was always exactly the same for every experiment and the second maximum at $n=1 \cdot 67$ was fairly steady, but the first maximum on different occasions varied, sometimes appearing at $n=1 \cdot 65$. In this case besides the above considerations there is an additional explanation of this. Rubens' curve shows that in the 
neighbourhood of $n=1.65$ a comparatively large change of refractive index involves only a sunall change in wave-length, and therefore the wave-lengths used at $n=1 \cdot 65$ differ only slightly from those at $n=1 \cdot 66$. With regard to the minimum a rather closer study of the region of this point was made by taking readings for the ranges of spectrum with centres at $n=1.665$ and $n=1.675$; both of these readings were larger than for $n=1.67$ showing that the actual minimum cannot be very far from $n=1 \cdot 67$. The wave-length corresponding to $n=1.67$ is not known, Rubens' readings only going as far as $n=1 \cdot 65$.

An absorption band is, however, known to exist in this region, and quartz is supposed to cut off all rays of wavelength less than $185 \mu \mu$. Wood (Physical Optics, p. 324) calculates the ultra-violet absorption band at $103 \mu \mu$. It seems likely that the electrical minimum at $n=1 \cdot 67$ is coincident with the absorption band in quartz, in which case the statement that quartz is opaque to rays less than $18 \tilde{a} \mu \mu$ in wave-length is erroneous, for the second maximum which was always well marked is beyond this limit. In any case it is highly improbable that for $n=1 \cdot 70$, at which the electrical effect was measurable, the wave-length should not be less than $185 \mu \mu$, for it is unlikely that from $n=1.65$ to $n=1.70$ the wave-length should not change by more than $15 \mu \mu$.

It is interesting to note that in Pfliger's research on the energy of the ultra-violet spectrum, conducted under very much the same conditions as the present electrical one, except that he used a fluorite prism, the energy commencing in the visible spectrum was very small until he reached $\lambda=190 \mu \mu$ when it rose to a very strongly marked maximum. This would coincide very nearly with the first maximum in the electrical curve. Too much stress should not, however, be laid upon this, as for reasons indicated previously there is not to be expected any simple relation between the energy of the spectrum and the electrical effect. It is probable that the electrical action is of the nature of a resonance effect in which case the energy would not be the primary factor though it would of course contribute. Except at the maximum there is no similarity between the energy curve of Pfligger and fig. 4. A final point of interest is that hitherto it was supposed that there was no electrical action in the visible spectrum, but with the sensitive methods of measurement used in this research a small effect was detected in the visible spectrum (which extended from $n=1.54$ to $n=1 \cdot 56$ ) though it was only two per cent. of the maximum effect. To make certain of the presence of this, a plate of glass was inserted 
300 Method of Reading Torsional Angle of Rotating Shaft.

to cut off any possible stray ultra-violet light but the small electrical effect still persisted.

In conclusion I should like to express my warmest thanks to Professor J. S. Townsend, in whose laboratory this research was conducted, and at whose suggestion it was undertaken, for assistance and encouragement al ways most readily given.

XXIX. An Optical Method of Reading the Torsional Angle of a Rotating Shaft. By F. J. Jervis-Syith, M.A., Oxon., F.R.S.**

$\mathrm{D}^{\mathrm{w}}$ URING certain experimental work in which it was necessary that the energy transmitted by a rotating shaft should be known, I devised the following method of reading the angle of twist of the shaft, and found that it gave good results. It is easily applied, and might be used for other similar purposes in the mechanical and physical laboratory. Clear readings can be taken from a pointer, moving over a circular dial which reflects light, while it rotates about a diameter. The optical principle involved is similar to that of the thanmatrope of Dr. Paris, in which on one side of a card the head of a man was painted, and on the other side a hat. When the card was rotated by means of twisted strings attached to the opposite edges of the card the head and hat appcared as one picture. The rationale of the experiment being that the picture of the head is retained by the organs of vision until the hat appears, the two separate impressions thus maling one picture. In my apparatus the reflecting dial, made of mirror glass, is fixed so that its plane is parallel with the axis of the shatt, or spiral spring, the angle of twist of which is to be measured. The mirror is perforated in the centre, and throngh the perforation the pivot which carries the pointer passes. The pointer is deflected through an angle proportional to that of the twist of the shaft by means of connecting links attached to the shaft and also to a sleeve fixed to the shaft.

A parallel beam of light is projected on to the mirror dial by means of two plano-convex lenses, as used in a projection lantern. The image of the pointer moving over the divided scale can either be viewed direct by reflexion, or, which is far more convenient, the image can be projected on to a screen by means of two achromatic lenses, and then it may be viewed simultaneously by several observers. This latter method of viewing the pointer is preferable to the

* Communicated by the Author. 